\title{
An Exploration of Problem Solving Skills in Science and Engineering
}

\author{
A.V. Murali \\ Software consultant
}

\begin{abstract}
Development of problem solving skills in science and engineering requires a conscious and sustained effort on the part of students. This is especially so in the case of mathematics, which requires logical thinking at every step. In this paper we investigate the essential ingredients of problem solving process, taking mathematics as an example. While some students love the challenge of solving difficult problems there are many who have fear of mathematics. To address this challenge we need to go to the very root of understanding the essential ingredients of the problem solving process. This paper serves 2 objectives: First, it aims to help students gain mastery over a subject by providing them a deeper understanding of the problem solving process. Secondly, it provides us a direction for developing an educational software tool with an assessment engine that can identify a student's level and his areas of weakness. Such a tool can in turn offer the student more practice in his areas of weakness thereby help him improve. With these objectives, the paper first identifies the essential ingredients of the problem solving process. Subsequently, it identifies in detail the types of tasks that are commonly encountered while solving math problems.
\end{abstract}

Keywords: Task types, Essential Ingredients, Problem solving skills, Assessment tool, Metacognition

\section{Introduction}

Problem solving is an essential aspect of all scientific and engineering disciplines. Students are assessed for their ability to solve problems as it gives us an indication of their level of understanding of the subject. In this paper we consider problem solving in mathematics for our investigation. The same ideas can be applied or extended to other science and engineering disciplines as well.

Many students have fear of mathematics. Fear has been identified as one of the major causes of math learning problems and learning disabilities. Other than fear, some of the common causes of mathematics learning disabilities have been identified as memory problems, attention problems, passive learning and lack of meta-cognitive thinking. Because math is difficult for them, math sessions are often an anxiety-ridden experience. While the best cure for math anxiety seems to be "success" how do we enable students achieve that?

\section{Literature Survey}

To address this problem, mathematicians and educationists have studied the process of solving mathematical problems [1]-[5]. They emphasized the importance of a systematic approach for solving problems. Georg Polya, an eminent mathematician said "If you can't solve a problem, then there is an easier problem you can solve: find it". He proposed 4 major principles that concern solving math problems. They are listed out here along with the key activities that go with them:

1) Understand the problem: Understand every word, rephrase the problem, represent it pictorially.

2)Devise a plan : Make a guess, make an orderly list, eliminate possibilities, use symmetry, consider special cases, look for a pattern, draw a picture, solve a simpler problem, work backward, use a formula, be creative.
3)Carry out the plan: This requires persistence and patience. Discard a plan and choose another only if it does not work after a serious attempt.

4)Check the result: Take time to reflect and look back at what you have done, what worked and what didn't. This would help in solving future problems.

These steps were elaborated in his famous book titled "How to solve it" [1].

Alan Schoenfeld, a well-known math educationist, observes that problem solving and discovery should go together [2][4]. He emphasizes on self-regulation during problem solving. He calls this ability of being able to 'choose the right action' during various phases of problem solving as 'control'. He further observes that the single most important thing for being able to solve extremely tough math problems is great control. He divides the problem solving procedure into following major steps:
1) Read
2) Analyze
3) Explore
4) Plan
5) Implement
6) Verify

In his experiments on novice as well as expert problem solvers, he found that novices spend more time on exploration than planning whereas expert problem solvers spend longer time in the planning step. Further, he emphasizes the importance of practice on a wide variety of problems and the role of metacognition, i.e., the knowledge and control of cognition.

\section{Problem Definition}

From the above discussion, it is clear that the problem at hand is the students' difficulties in solving math problems. We need to find a way of solving this issue so that the fear of 


\section{International Journal of Science and Research (IJSR) \\ ISSN (Online): 2319-7064}

Index Copernicus Value (2013): 6.14 | Impact Factor (2015): 6.391

mathematics can be dispelled. The same techniques can then be applied to other areas of science and engineering.

\section{Methodology and Approach}

A detailed study of solutions to several hundreds of mathematical problems, at high school and higher secondary levels, has been carried out by the author. The study has led to identification of the essential ingredients for solving problems, on one hand. On the other hand, it has led to identification of a number of 'task types' that are generally carried out while solving problems. It is expected that this investigation would give a clear direction and a preparatory framework to students who aspire to develop their problem solving skills. This would also help us in building an assessment tool that can find out a student's weak areas and provide further support by offering focused practice sessions.

\subsection{The Essential Ingredients}

To get over the difficulty in solving problems we need to first understand what exactly constitutes problem solving skill. We need to ask what is it that we need to do to be a good problem solver. If we carefully analyze the process of problem solving in mathematics, we realize that the following ingredients are essential to be a successful problem solver:

1)Practice (memory about axioms, results and reasoning)

2)Motivation (drive)

3)Observation (attention)

4) Visualization (of logical connections) and Pattern recognition

5)Planning (method)

6)Exploration and Control (convergent-divergent thinking, belief revision and maneuvering)

While the first three ingredients listed above are the fundamental requirements to be a good problem solver, getting a student to practice, feel motivated and pay attention requires success at problem solving. This may seem to be a vicious cycle but it is possible to break it by adopting a gradual approach. Small successes encourage a student to go deeper and attempt tougher problems. Students should first be given simpler problems that require them to apply just one or two axioms. Once he shows mastery in it he could be exposed to more difficult problems that require one to visualize the logical sequence of steps, plan the steps and apply heuristics. He should be taught to understand the problem statement thoroughly, know how to rephrase it, find the linkages between the elements of the given problem and the solving techniques that are at his disposal. He should be encouraged to focus on the mechanics of the problem solving process, visualization of steps, planning and the maneuvering that is required to wade through the maze of logical paths.

The process of solving difficult problems involves a combination of focused and divergent thinking. Exploration that we do during the process of solving a problem leads us to many useless branches or lines of reasoning which may have to be abandoned before arriving at the right line of reasoning. An expert problem solver would not only be good at pruning such useless lines of reasoning but would also have a natural tendency to consider variations in the premises or intermediate conditions that arise when he is solving a problem. He would device a plan and then go about exploring it further rather than going about doing a blind exploration. An expert employs a variety of heuristics that mathematicians have devised in order to take the right line of reasoning. In the course of solving a problem, if he finds that his line of reasoning is not very promising, he would either abandon that and try an alternate course or make some variations to the given premises and see whether he can solve a simpler problem with less constraints and then add more constraints to it to solve the given problem.

\subsection{The Task Types}

While the essential ingredients that were listed in the previous section form the fundamental principles that one needs to imbibe to be a good problem solver, we also need an understanding of the types of tasks that one needs to carry out at every step in the process of solving a problem. The recognition of task types gives the student a better grasp over the problem solving process and allows him to exercise his metacognitive thinking. Here we aim to elaborate on these task types further.

Math students can recognize that some of the common tasks that are carried out while solving a math problem are, making a construction (as in geometry), establishing or refuting some assertion, finding properties of certain entities, finding or establishing relationships between entities and determining some unknowns.

An extensive analysis of hundreds of math problems at the high school level up to the level of competitive examinations like IIT JEE, has shown that the task types that we encounter while solving math problems fall into one of the 50 types that are listed here. The areas of mathematics that have been investigated are algebra, geometry, trigonometry, calculus, probability and statistics. The occurrence of a task type in a specific problem depends on the type of the problem. Difficult problems typically exhibit many of these task types. The key task types that have been identified are shown in capitals to emphasize their importance. It is recommended that students consciously recognize these task types when they are solving math problems.

1. REPHRASE THE PROBLEM STATEMENT: Understand the problem, analyze its parts and formulate or represent the problem statement in an appropriate mathematical form

2. FIND AN INSTANCE of an assertion or an operation or an operand.

3. JUSTIFY OR REFUTE a statement with an example.

4. DEVISE A PLAN.

5. CONSTRUCT AN ENTITY OR EXTEND it.

6. RECOGNIZE THE TYPE an entity belongs to.

7. IDENTIFY ASSOCIATIONS between entities.

8. CHECK APPLICABILITY of a rule or theorem or technique.

9. RECOGNIZE A CRITERION for applying a known result. 


\section{International Journal of Science and Research (IJSR) \\ ISSN (Online): 2319-7064}

Index Copernicus Value (2013): 6.14 | Impact Factor (2015): 6.391

10. APPLY A TECHNIQUE: Some examples of techniques are substitution, rearrangement or regrouping, separation or combination, elimination, rationalization, expansion, reduction or simplification, induction, a pair of cancelling operations, taking componendo-dividendo and transformation.

11. APPLY A RESULT: The result could be a theorem, an axiom, a known result or a property.

12. APPLY A RULE.

13. DETERMINE THE DOMAIN of a variable.

14. NARROW THE DOMAIN of a variable.

15. FIND THE RANGE OF A FUNCTION.

16. RECOGNIZE A PATTERN.

17. COMBINE RESULTS.

18. RECOGNIZE A PROPERTY.

19. ARRIVE AT A RELATIONSHIP.

20. REPRESENT AN EXPRESSION BY A SYMBOL.

21. CHOOSE A REASONING MODE: Forward or backward chaining or Hybrid Mode of reasoning. Forward chaining means to start from premise to goal while backward chaining means to start from goal to premise.

22. RESTATE a Condition or an Expression or a Relation in a different form.

23. ENUMERATE the sample space with or without constraints.

24. REDUCE OR CONVERT TO (or Closer to) A STANDARD FORM OR REQUIRED FORM.

25. SET SUBGOALS OR MILESTONES. i.e., create subproblems, decompose a problem and use divide and conquer strategy.

26. ESTABLISH A SUB-GOAL

27. CONSIDER A SPECIAL CASE.

28. GO FROM SPECIAL CASE TO A GENERAL CASE.

29. USE A LEMMA.

30. ARRIVE AT A COROLLARY.

31. CHANGE OR SIMPLIFY

TEMPORARILY.

32. CONSIDER GIVEN PREMISES IN TOTALITY.

33. CLASSIFY, ARRANGE OR ORGANIZE a set of values or entities into classes.

34. DETERMINE THE BEHAVIOUR or characteristic (e.g., that of a function).

35. COMPARE two or more entities or inferences.

36. CONTRAST OR DIFFERENTIATE two or more entities or inferences

37. GENERATE EXAMPLE OR COUNTEREXAMPLE.

38. COMPUTE OR EVALUATE an algebraic expression.

39. ESTIMATE value, behavior or form. Arrive at an approximation.

40. SHIFT PERSPECTIVE OR LEVEL OF ABSTRACTION. This is a very powerful technique that only expert problem solvers would be able to employ, as it comes out of experience and a deeper understanding of the logical connections. It involves identifying a remotely connected concept or algebraic or geometric form and using it to solve a given problem.

41. INTERPRET RESULT.

42. REPRESENT OR SKETCH result pictorially.

43. SEARCH FOR VALUES OR VALID EXPRESSIONS either systematically or by trial and error.

44. MAKE AN ASSUMPTION.

45. VALIDATE OR INVALIDATE ASSUMPTION.
46. IDENTIFY DEPENDENCIES. Dependencies may be hidden or visible or implied or stated.

47. VARY A VARIABLE'S VALUE.

48. UPDATE DEPENDENT VARIABLE VALUES.

49. EXTEND OR GENERALIZE A RESULT.

50. ARRIVE AT A COROLLARY.

The author recognizes that it would have been desirable to illustrate the above list of task types with one or two examples of solutions to problems. For want of space, no descriptions or illustrations have been provided here. But a reader would be able to recognize these task types by taking a solution to a math problem and trying to identify the task type that each step in the solution belongs to.

\section{Discussion}

A formal knowledge and understanding of the task types like this helps students in more than one way. First, a student gets to know what all tools are available to him for solving a given problem. Given a problem he would apply them consciously. It demystifies the math problem solving process. Secondly, it helps math teachers enrich their problem solving sessions by explicitly telling the students what is being done at every step.

In addition to this, it helps us in developing a math assessment software that gauges a student's areas of strengths and weakness at a very fine level of granularity. To achieve this objective all we need to have is a knowledge base of solutions to a wide variety of math problems along with solutions to each of them. Every step in the solution shall be assigned the appropriate task type based on what is being done at that step. If a step requires identification of a pattern then the task type 16 from the above list would be assigned to that step. The software tool would pose a math problem and let the student solve it interactively step-by-step. As the software takes the student through the steps in the solution, it asks the student to choose what he would do next from a set of available options. If a student is weak in recognizing an algebraic pattern, he would not be able to proceed with that step which requires that task type to be identified before he could proceed to the next step. This way it helps teachers identify the areas a student is weak at and focus on it more. The software itself can offer more problems requiring application of such tasks that a student is weak at and thereby help him overcome his weaknesses.

\section{Future Scope}

The author intends to take this investigation further, apply it to school curriculum and study its impact on the problem solving skills of students at high school and higher secondary levels. While the above study focusses mainly on mathematics, the author believes that the same ideas can be transferred to other areas of science and engineering. Further, it is planned to develop an assessment tool that would incorporate the above ideas to identify a student's areas of strengths and weaknesses at a fine level of granularity. 


\section{Conclusion}

We have investigated the essential ingredients that are required to be a good problem solver. We have identified the types of tasks that we carry out while solving any given problem in mathematics. It is hoped that an understanding of these essentials and the task types would give a student a better grasp on the subject of mathematics and help him improve his problem solving skills. While the discussion has been primarily confined to mathematics, the same can be applied to other branches of science and engineering, with some minor enhancements. One such enhancement could be addition of 'model creation' as a task type. This is an important step in many problems in physics where we construct a free body diagram. It is also hoped that this investigation would help us in building an assessment tool that can identify a student's strengths and weaknesses and hence help him focus on his weak areas.

\section{References}

[1] G. Polya, How to Solve It: A New Aspect "of Mathematical Method (Princeton Science Library), Princeton, 2014.

[2] A.H. Schoenfeld, Mathematical Problem Solving, Academic Press, California, 1985.

[3] A.H. Schoenfeld, How We Think: A Theory of GoalOriented Decision Making and its Educational Applications (Studies in Mathematical Thinking and Learning Series), Routledge, California, 2010.

[4] A.H. Schoenfeld, A.H. Sloane, Mathematical Thinking and Problem Solving (Studies in Mathematical Thinking and Learning Series), Routledge, California, 1994.

[5] T. Tao, Solving Mathematical Problems: A Personal Perspective 1st Edition, Oxford University Press, Oxford, 2006.

\section{Author Profile}

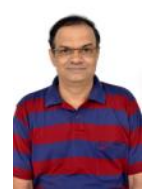

A.V. Murali received his B.Tech and M.Tech degrees in Metallurgical Engineering from IIT Madras. He has got an extensive work experience of over 25 years in diverse software application domains. His areas of work are primarily centered on applied research and systems implementation. His areas of research interest include software engineering, data mining and text mining, mathematical problem solving and game based learning. 